\title{
Multilatéralisme créatif : une collaboration renforcée, pour tous
}

Dernière mise à jour : 28 mars 2018

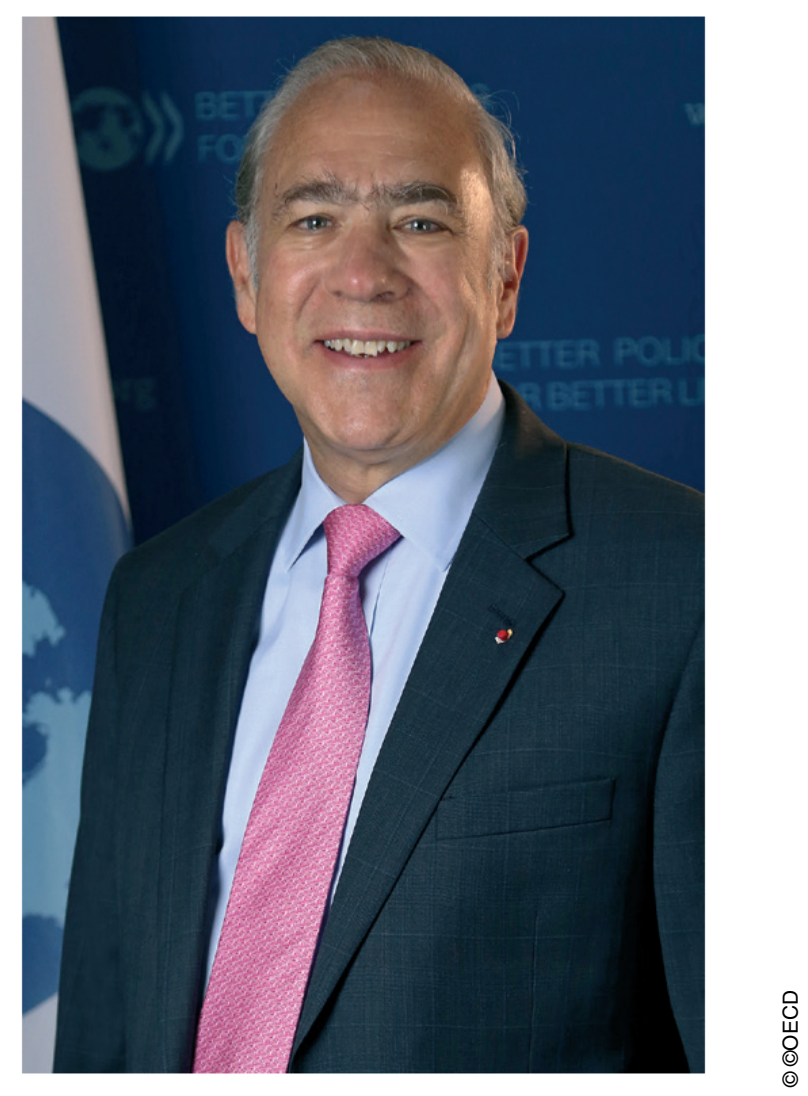

Multilatéralisme créatif : une collaboration renforcée, pour tous

Le système international a atteint un point critique, confronté à une croissance économique mondiale en berne, des inégalités croissantes, et une remise en question de l'ordre mondial fondé sur des règles, garant de la paix et de la prospérité dans le monde depuis des décennies. De nombreux gouvernements s'efforcent de réajuster leur engagement mondial et de faire ce qui est en leur pouvoir pour que le monde reste ouvert et progressiste.* 
Cinq pays aux profils assez différents - l'Australie, la République de Corée, l'Indonésie, le Mexique et la Turquie — sont à la pointe de ce mouvement et se sont regroupés pour former un partenariat innovant, les « MIKTA ». Bien qu'informel, ce groupement pourrait s'ériger en exemple du type de diplomatie moderne et de coopération internationale dynamiques actuellement nécessaires pour dépasser les clivages et favoriser un système multilatéral.

Le groupement des MIKTA est unique en son genre : nous sommes cinq partenaires représentatifs de la diversité de la communauté internationale, tirant nos idées et perspectives de nos traditions régionales, culturelles, religieuses et historiques respectives. La force des intérêts qui nous rassemblent - un engagement en faveur d'un ordre mondial fondé sur des règles et la défense d'institutions internationales au service de tous — transcende nos différences. Ce qui compte, c'est ce qui nous rapproche.

Pareille démarche est plus importante aujourd'hui qu'elle ne l'a jamais été. L'intérêt du système commercial mondial et d'autres enceintes et initiatives multilatérales est contesté, et violemment remis en cause par ceux-là mêmes qui ont œuvré à leur mise en place. L'économie mondiale connaît actuellement des mutations profondes et durables, qui offrent des opportunités à beaucoup mais apportent également leur lot de difficultés à d'autres. D'autre part, une nouvelle forme de scepticisme se fait jour. Nombreux sont ceux qui estiment que la mondialisation ne leur correspond pas ou ne répond pas à leurs besoins. Les organisations internationales, dont l'OCDE, qui ont favorisé la création du cadre sous-tendant l'ordre mondial fondé sur des règles et garant de paix et de prospérité, sont aujourd'hui accusées par certains d'être une partie du problème plutôt que de sa solution.

Dans ce climat d'incertitude, le système multilatéral dans son ensemble a besoin de partenariats constructifs pour rassembler les pays et trouver des solutions communes à des problèmes complexes et délicats. Voilà la mission que les MIKTA se sont fixée dans le cadre de ce partenariat. En tant que rassembleurs et bâtisseurs de passerelles, nous cherchons à faciliter le dialogue et à contribuer de manière constructive au débat au sein de plusieurs instances. Ainsi, nous pourrons faire du système multilatéral une solution bénéfique au plus grand nombre.

Par exemple, notre Atelier sur le commerce et l'investissement, hébergé par l'Organisation mondiale du commerce, a permis, par ses débats, de faire naître des propositions concrètes sur les domaines dans lesquels l'OMC pourrait apporter une valeur ajoutée, notamment sur la cohérence des politiques commerciales et d'investissement, et la facilitation de l'investissement. Cette initiative montre bien ce que peuvent accomplir les économies développées et en développement lorsqu'ils travaillent ensemble. À cette occasion, comme en tant d'autres, la diversité du groupe MIKTA a fait sa force. 
Dans le cadre de nos travaux en faveur d'un système multilatéral, nous soutenons également les efforts déployés pour améliorer le fonctionnement et la gouvernance des organisations internationales. Nous nous employons notamment à actualiser la gouvernance internationale en matière d'énergie, pour qu'elle tienne mieux compte des besoins des pays émergents et en développement. Cet état d'esprit transparaît dans l'ambitieux programme de modernisation sur deux ans de l'Agence internationale de l'énergie.

Différents, mais unis par une vision commune : voilà ce qui donne au groupe des MIKTA sa force, et ce qui peut en faire un modèle pour d'autres pays souhaitant travailler ensemble pour soutenir la paix et la prospérité mondiales. Nos différences rendent ce qui nous rapproche encore plus précieux : l'ordre mondial fondé sur des règles, dont nous dépendons tous.

*Par les ambassadeurs du Mexique, de la Corée, de la Turquie et de l'Australie auprès de l'OCDE : Mme Monica Aspe, Mexique ; M. Jong-Won Yoon, Corée ; M. Erdem Başçı, Turquie ; M. Brian Pontifex, Australie ; et l'Ambassadeur de l'Indonésie auprès de la France, M. Hotmangaradja Pandjaitan.

Voirwww.mikta.org

@L'Observateur de l'OCDE, mars 2018 